le Silure se rencontre maintenant clans la plupart des étangs de Hongrie, entrant dans la composition du peuplement pour une proportion qui varie de I I/2 à 5 pour cent. Cependant, insistons sur ce point quie la fraye et l'ạlevinage ne réussissent pas toujours ni partout. Dé là le prix élevé des sujets de un été $(0,10$ à 0,50 pengö la pièce) .

Comme il a été dit, l'éleveur qui á obtenu le meilleur succès est M. Z. Concrus A Ugra, dans le Ciomitat de Bihar, sa production annuelle de Silures âgés dẹ 3-4 étés s'élève à 35 -40 quintaux ; elle ạtteint assez régulièrement la même importancè à Kelabia, dans̄ le Comitat de Bács. Le nombre-des spécimens de I été qui seront dispónibles pour la vente à l'automne prochain est évalué à. 10-12.000.

Toutefois, ce pisciculteur d'avant-garde ne jouit pluș, comme précédemment, d'une sorte de monopole. A Tată à Hortobăgy et àlleứs j̣ est rare, maintenant, que l'alevinàge ne réussisse pas ; on obtient donc sur place un empoissonnage dont l'excédent se place facilement avec sérieux bénéfice. En outre, le Comte $F$. Esterhazy est à même de livrer aú commerce après cháque camṕagne, de 30 à 50 quintaux de poíssons de consommation, vieux de 3 ans ou plus.

On peut considérer la technique de l'élevage du grand Silure comme actuellement au point. Peut-êtré les carpiculteurs d'autres pays que la Hongrie trouveraient-ils aussi avantage à introduire dans leurs étangs celte excellente espèce., à tịtre complémentaire.

\title{
Bibliograpuie
}

- I. - RÉpassy Miklós. - Edesvizi halászạt ès halgrázdaság. - Budapest. - $\mathrm{r}^{\mathrm{re}}$ édition, $1908 ;-\mathrm{2}^{\dot{\mathrm{e}}}$ édition, rgi4, p. 203.

2. - B - Gyakorlati tapasztalatók tògazdaságok köréböl. - Halászal, XXIII ; $\div$ 1922, p. 43 .

3. - Bacsá Béla : - A lesö harcsa tenyésztése. - Halászat, XX V̈III ; 1927, p. 81 :

4. - Conchus Zoltán : - Ragadozò halak a tógazdastágban ; -

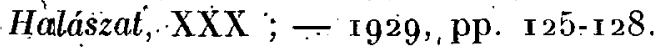

\section{LA MORTALITÉ ESTIVALE DES CARPES SES CAUSES, SES REMÈDES}

\author{
Par M. Michel LHÉRITIER
}

Plsciculteur à Ambazac (Haute-Vienne).

Tous les carpiculteurs des régions lempérées, particulièrement ceux dont les petits étangś ne sont pas ạlimentés par des ruịsseaux ou dẹs sourcess, ont constaté qu à certaines époques de l'année, notamment durant la canicule, une quantité souvent considérạble de Cárpẹs mourạit, sans cause 
apparente el d'une manière si rapide quion pouvait croire à un emppisonnement ou à une épidémie foudroyante.

Cependant, l'autopsie des victimes, et mème l'analyse des eaux, n'ont jamais permis de déterminer aveç certitude la cause de ces troublants phénomènes.

Ainsi, il y a quelques années, chez l'un de nos plus sympathiques collègues, qui est, en même temps, l'un des plus habiles et des plus réputés carpiculteurs de notre époque, un accident de cetle nature se manifesta dans une séric de trois étangs de pose se faisant suite, avec cette particularité surprenante que la mortalité, considérable dans les deux étangrs extrèmes, fut nulle dans celui du milieu (1).

La chose fut si soudaine qu'aux yeux de tous l'empoisonnement seul pouvait avoir causé de tels ravages.

Bien que notre collègue soit un bon maître, certain du dévouement dẹ son personnel et assuré de la sincère estime de ses voisins, il crut à l'empoisonnement et fit prélever des échantillons de l'eau des étangs dans lesquels mouraient les poissons. Il les expédia d'urgence aux laboratoires connus de Paris, Grenoble, etc;, puis porta plainte contre $\mathbf{X}$.

J'avoue avoir attendu avec curiosilé le résultat des analyses, mais n'avoir pas été surpris qu’il fut négatif. Cependant, j'espérais que, parmi les vieux pratiçiens de l'Union Nationale des Syndicats de l'Etang, il s'en trouverait pouvant donner une explication plausible du phénomène, montrant que la Science pure ne peut se passeir de l'expérience. Quelques mois plus tard, le Bulletin de l'U.N.S.E. restant muet sur cette question, j'écrivis à notre collègue pour l'assurer que son entourage n'avait pas démérité de.sa confiance, car l'empoisonnement réel avait des causes absolument naturelles que je lui indiquais, ainsi que les moyens de parer au danger en cas de récidive.

Jè regrette que ces renseignements n'aient pas été transmis au Bulletin de l'U.N.S.E., cela aurait pu ètre utile à nos collègues et aux savants qui s'intéressent avec tant de dévouement à l'hydrobiologie, car ces disciples el continuateurs du grand Büfon n'ont certainement pas oublié les paroles du Maître : - "Messieurs, recherchons des faits pour avoir des idées."

Il est indéniable que, dans le cas que nous venons de rappeler, les poissons sont morts empoisonnés ou, plus exactement, asphyxiés par l'hydrłogène sulfuré qui existe en plus ou moins grande abondance dans la vase accúmulée au fond des étangs. Sa présence est décelée par une odeur sui generis au moment où se produisent les accidents ou quand, par siphonage ou manouvre de bonde, on soulire ou laisse écouler l'eau du fond.

Des esprits superficiels s'étonneront que, dans ces conditions, les poissons puissent vivre dans les étangs d'eau stagnante, mais, s'ils veulent bien lire

(1) Bulletin de l'Union Nationalé des syndiculs de l'Llang; - Lome 15; $11^{\circ} 39$, 1. 1498 . - Imprimeric du Loiret, Orléans. 
attentivement le, clair exposé de notre collègue, ils comprendront facilement pourquoi j'affirme cque l'empoisonnement constaté a des causes naturelles, et comment se prduisent toujours les accidents' de ce genre.

Tout d'alord, écrit le sinistré; “ j’ai, devant la grillè de là maison, trois bassins rangés :-Pigeonnior ; - Milieu;-Cọmpost.

"Pigeonnier avait reç le groupe “ Ḣnscri " exposénourrain à Paris et de toute beauté : I 500 mètres carrés.

"Milieu riavait rien d’intéressant : r.ooo mètres'carrés.

"Compost : deux groùpes "Meugnior " cuir ; environ 2.50o mètres carrés.

'c Pigeonnier et Compost avaient posé Lè is Août áu soir, tout s'y comportait fort bièn. L'eau était coupée dejuis trois semäines et, idu reste, ne coulait plus. La charge en fẹulles était manifestement très inférieure à celle des années précédentes, les géniteurs étant dạrs leur troisième année seulèment:

“Le 19 Aồt, à l'aube, Carpes et carpillonnes pipaient désespérément dans Pigeonnier et Compost : elles se trainaient sans force la gueule hors de l'eau ; on les pöuvait prendre à la män. L'eau de ces bassins élait devenue beaucoup plus cháude que celle de Milicu où rien ne se manifestait. Dane Pigeonnier on voyait plusièurs feuilles crevées au fond. 'D'autres, d'un. sáut épileptiqúe, venaient crever sur là rive. Deux géniteurs expiraient au bord. Je saisis le troisième et le mis dan's Milieu où il se rếveilla vite. J'eus, comme mes hommes, l'impression éviden lé du poison. Compost, plus grand, se défendait mieux, voilà tout:

"Je m’en fus quérir le brigadier, lavai i 2 litres el prélevai l'eau. Stupidement, je la pris là où les Carpes venaient mourir alors qu'elles y' cherchaient une eau moins contaminée... On ne pense pas à tout.... Mais j'eus. une meilleure jdée : je lâchai la bonde.

"Vers 8 heures, aucune Carpe ne crevait plus. La bonde levee avait suffi à Pigieonnier où je ne pouvais envoyer d'eau aussitôt. A Compost, je pus renouveler l'eá; il n'y eut pas de perte. Leffet du poison, probablement jeté à la bonde avant l'aube, n avait duré que 4 heures environ: Comme il y avait des morts, je pêchai Pigeonnier au couchè du soleil, sanis une perte .nouvelle. "

Les mots en italique dans le texte-ci-dessus atticeront l'attentión du lecteur désireux de suivre le raisonnement d'un auleur que son humble origrinè n'a pas préparé aux exposés sçientifiques, mais dont les arguments s'appuient sur des faits qui paraissent de nature à éclairér la discussion.

Durant plus de trente années de pratique piscicole, dont quinze de salmoniculture, j'ai eu à lutter maintes fois contre'des accidents de ce genre ; aussi pus-je en déterminer la cause essentielle ct trouver les remèdes à lui opposer. Mais, comme daus tous les cas semblables, l'application des meilleurs remèdes est, le plus souvent, trop tardive el prévenir est.infiniment plus facile que guérir. 
Quand on entrepose des poissons dans -un bassiñ ou un appareil de dimensions insuffisantes, on voit, peu de temps après, les animaux manifester leur malaise en venant humer l'air à la surface de l'eau ; on dit qu'ils " pipent ". Si l'on ne vient pas̀ rapidement à leur secours en remuant brusquement le récipient, en brassant vivement l'eau, ou en y en versant d'autre, on voit les malheureuses bêtes s'agiter désespérément, leụrs lèvres se décolorent, leur robe entière pâlit ; puiś élles coulent à fond, ventre en l'air.

On sait, en effet, que la quantité d'oxygène dissous dans l'eau est peu considérable et que les échanges respiratoires des poissons dégagent de l'acide carbonique qui rend bientôt le miliẹ inhabitable. Or, l'été, quand la température s'élève, la quantité d'oxygène dissous va diminuant, alors que l'activité respiratoire des poissons croît. On les voit dọc en mauvaịse posture. Mais il y a pire encore. Sous l'influence de la chaleur solaire, la matière, organique contenue dans la vase dégage dẹs gaz pestilentiels, tẹl l'hydrogène sulfuré dont une quantité infinitésimale provoque, chéz les poissons, des mouvements épileptiformes et les fait périr en masse dans des convulsions caractéristiques.

Ce gaz, plus lourd que l'air puisqu'il stationne ordinairement dans les couches profondes, s'élevant près de la surface sous l'influence de la température, asphyxie les poissons quii, affolés, tentent vạinement de le fuịr. Ainsi que l'expose notre collègue : - " dạns Pigeonniên on voyạit plusieurs fẹilles crevées au fond, d'autres, d'un saut épileptique, venaiẹnt crever sur la rive ". Ceci prouve que les poissons tentaient de fuị la nappe de gaz asphyxiants.

Un autre fait est significatif, c'est que le troisième géniteur, mị dans Milieu, se remit très vite. Preuve encore que la suppression de la cause supprimait les effets.

L'idée de lâcher la bonde fut géniale, car l'eau s'en allant à flots emportait le gaz noctif, si bien que : - "vers 8 heures, aucune Carpe né crevait plus. 's.

Quant à Milieu, s'il fut indemne d'accidents, l'explication s'en trouve dans cette phrase : - "L'eau de ces bassins (Pigeonnier et Compost) était devenue beaucoup plus chaude que celle de Milieu. " Cette observation justifie l'opinion précédemment émise que les dégagements de gaz nocifs se produisent en proportión directe de l'élévation de température de l'eau.

Enfin, dans une lettre suivant l'exposé de notre Collègue et à lui envoyée par un des chimistes consultés, on peut lire :- "Je crois que la mortalité constatée brusquement lé matin du ig Août, dans deux réserves indépendantes, mais voisines, que j'ai eu l'occasion de voir, ne peut guère s'expliquer sans l'intervention d'un élément nocif introduit dans ces réserves. "

Je considère que celte opinion est erronée.

Cet élếment a eu une action immédiate intense, mais éphémère, ne laissant plus de trace décelable après quelque témps, ainși que 1:a montré 
l'analyse chimique qui, pratiquéé seulement plúsieurs jours après l'accident, n'a pas permis de trouver d'élément minéral toxique. Il ne poivait en être autrement.

"Cependant, - continue la lettre, - l'éa quii noüs á été àdrèssée au láboratoire avait une odeur assez prononcée d'hydrogènè sulfurué; indicè, soit de corruption putride, soit de la présence-d'un sulfure soluble dans l'eau. Son action sur les poissons salmonides était sidérante. Lés Cyprinides la supportaient un peu plus longtemps, mais étaient rapidement incommodés. Au bout de quelques heires de contact àvè l'air; l'élément toxique de l'eau devenait inactif ou avait dispáru, sảns doùtè pảr oxyda: tion, ce qui explique le résultat de l'ànalyse chióniqué. "

Personnèllement, je considère que l'odeur assez phononicée d’hydrogène sulfüré était un indice suffisant pour fixer l'opinion d'un praticien:

Pour faciliter les recherches des chimistes et biologistes; je puis préciser que les cinq ou six accidents du genre de celui qui vient d'être rappelé, que j'ai constatés depuis I9o\%, se sont toujours produits en Août et à l'aurore, ce qui me fait supposer que la fraîcheur de l'air ambiant provoque l'ascension du gaz nocif vers la surface de l'eau restée plus chaude que l'air.

Une fois; cependant, semblable phénomène s'est produit lors d'une pêche d'étang exécutée tardivement en Mai, sur des carpillons de 2 étés très forts et vigoureux, rassemblée dans une fosse alimentée avec de l'eau assez claire-sortant par la bonde, mais coulant sur la vase chauffée par un soleil ardent. Ces carpillón's crevèrent en masse.

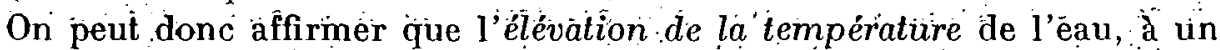
certain degné, variant ave cla souillure du fond, provoque le dégagement dë gaz toxíqueses capàbles de faire périr rapidement toute la pöpulatión d'un étang.

Je crois devoir ajouter que j'ai égàlement observé des empoisonnement frappant les Truites de rivière en eaux réputées assez pures, telles celles de la Creuse chantée par George SAND. Quand ce phénomène s'y pròduit, les vieux pêcheurs disent que les Truitês " rebouillissent ", car, luttant contre l'asphyxie, elles ont des mouvements dẹ́sordonnés, convulsifs, que nos campagnards comparent à ceux des légumes dans la marmite en ébullition.

Ges accidents se produisant toujours en Aout et durant les périodes où dominent'les vents secs de l'Est ou du Sü-Est; il est à croire que; pour tous les êtres vivants, la sécheresse est'beaucoup plus nuisible que l'humidité, car la première engendre la mort, tandis cque la seconde est; incontestablement, une source de viẹ.

De ce qui précède; on peut conclure que la mortalité estivâle des Carpes est surtoút causée par les gaz délétères qui, sous l'influence de la chaleur, se dégagent de la vase dès étangs; alors que l'élévation de température dè l'eau a réduit considérablement la quantité d'oxygène dissous, tandis qu'elle accroît l'activité respiratoire des poissons. 
La cause du mal étant reconnue, voici, maintenant, le remède à Iui appliquer.

Quand on s'aperçoit que les poissons d'une pièce d'eau quelconque donnent des signes de malaise, notamment en venant piper à la surface, s'obstinant à y-revenir après cqu'on les en a chassés, qu'ils s'agitent avec des mouvements épileptiformes, que leurs lèvres se décolorent, il faut, de toute urgence, leur donner l'air dont ils ont besoin. On y parviént, soit en brassant yivement l'eau au moyen de pelles, s il s'agit d'un bassin de peu d'étendue; d'une pompe à purin ou d'autres appareils mécaniques; s'il s'agit d'un étang. Mais, dès qu'on aura obtenu une amélioration dans l'état des poissons, on complétera le trạitement en désintoxiquant le milieu à l'aide d'une forte solution de permanganate de potasse. Une expénience, déjà longue, hélas! m'a mainles fois montré les merveilleux effets de ce produit.

La solution une fois répandue, ou bien les cristaux de permanganate jetés à la volée, on continuera quelque temps le brassage de l'eau et on ne tardera pas à constater qu'une odeur agréable se dégage de cette eau auparavant nauséabonde; les poissons reprennent leurs colorations et allures habituelles et, finalement, regagnent le fond.

11 est évident que si l'installation permet d'envoyer dans. l'étang contaminé l'eau d'une rivière ou celle provenant de la surface d'un étang voisin, on s'empressera de le faire.

Comme mesure préventive, l'assec hivernal et le chaulage énergique du sol ; puis, en Juillet-Aon̂t, de légerers poudrages de chaux pulvérisée sont à recommander.

Il m'est apparu récemment que la promenade en bateau actionné par un propulseur aérien placé à l'arrière était ü moyen amusant et pratique d'aérer rapidement une grande étendue d'eau.

\section{ERR ATA}

Deux fautes d'impression sont à corriger dans le précédent Bulletin.

A la page 249 , dans l'article sur "La Carpe limousine et la Station d'alevinage de Vauguenige ", la dix-huitième ligne est à reporter en bas de page.'

A la page 259 , dans l'article sur "L'action de l'électricité sur le pọisson et la technique des grilles électriques ", la formule (5) est à rectifier comme suit :

$$
\mathrm{R}=317 \cdot \operatorname{In} \frac{\operatorname{Sin} \pi(a j b+r / b)}{\operatorname{Sin} \pi \cdot r b}
$$

